\title{
ESTUDO DA MORFOLOGIA VASCULAR DO LEITO PLACENTÁRIO NA HIPERTENSÃO ARTERIAL CRÔNICA
}

\author{
Francisco lázaro Pereira de Sousa*, Nelson Sass, luiz Camano, João Norberto Stávale, Maria Rita de Sousa Mesquita, Eder Viana de Souza, \\ Fábio Roberto de Oliveira, Márcia Marcelino Souza Ishigal \\ Trabalho realizado no Serviço de Obstetricia do Hospital Guilherme Álvaro, Faculdade de Ciências Médicas de Santos - UNILUS, Santos, SP
}

\section{*Correspondência:}

Av. Presidente Wilson, $n^{\circ} 40$

- apto. 404 - Gonzaga

Santos - SP

CEP $11065-200$

\begin{abstract}
RESUMO
OвJEтIVo. Analisar os padrões histopatológicos das artérias do leito placentário em gestações complicadas por hipertensão arterial crônica (HAC) comparando-os com o de grávidas normais.

Métodos. Biópsias de leito placentário, segundo técnica de Robertson et al.', foram realizadas em pacientes com idade gestacional igual ou superior a 28 semanas, submetidas a cesárea, após a dequitação. $O$ grupo controle (GC) foi composto por 25 pacientes normais e o grupo de estudo composto por 13 pacientes com HAC leve (HL), II pacientes com HAC moderada $(H M)$ e II pacientes com HAC grave (HG). As classes de hipertensão foram consideradas leve (PAD 90 - 100 $\mathrm{mmHg}$ ), moderada (PAD $100 \mid-110 \mathrm{mmHg}$ ) e grave (PAD $110 \mathrm{mmHg}$ ). Os padrões histológicos das artérias espiraladas foram classificados em: padrão inalterado, modificações fisiológicas, desorganização da camada média, alterações hiperplásicas, necrose e aterose aguda.

Resultados. I) Os achados anormais foram predominantes no grupo de hipertensas, sendo mais freqüente nos grupos HM e HG. 2) $\mathrm{O}$ achado anormal mais prevalente foi a desorganização da camada média, com distribuição semelhante nos grupos HM e HG. 3) Os padrões normais ocorreram nos grupos GC e HL, com distribuição semelhante entre si.
\end{abstract}

UnITERMOS: Hipertensão arterial crônica. Leito placentário. Gravidez.

\section{INTRODUÇÃO}

A hipertensão arterial crônica (HAC) intercorrente na gestação, isolada ou associada à pré-eclâmpsia, representa intercorrência clínica relacionada com elevado risco de morbidade materna e perinatal.

Em nosso meio, apesar de não existirem estatísticas atualizadas, estima-se a presença de HAC em 6,6\% das mulheres inseridas no mercado de trabalho da Grande São Paulo, ${ }^{2}$ sendo estes números semelhantes àqueles registrados nos EUA para mulheres com 18 a 39 $a^{2}{ }^{3}$. Levando-se em conta que atualmente muitas mulheres planejam sua gestaçãa após sua terceira década de vida, é possível afirmar que a presença de HAC na gestação seja um fenômeno progressivamente mais freqüente $e^{4,5,6}$.

A circulação útero-placentária em gestantes portadoras de HAC pode ser prejudicada em graus variáveis, em função das modificações da microcirculação, mediadas por distúrbios funcionais, tais como ocorrem na pré-eclâmpsia ou decorrentes de lesões vasculares crônicas, resultando em queda expressiva da perfusão tecidual. Como resultante, ocorrem na placenta isquemia e infartos, muitas vezes em grande extensão, determinando insuficiência funcional e dificuldades para suprir as necessidades para o adequado desenvolvimento fetal em face da restrição da oferta de oxigênio e nutrientes, além de facilitar a ocorrência de eventos agudos como o descolamento prematuro da placenta.
As bases fisiopatológicas atualmente conhecidas explicam em parte os resultados clínicos observados, uma vez que gestantes hipertensas com expressão clínica leve freqüentemente apresentam desempenho perinatal muito próximo da população normal. Tal constatação permite supor que, entre gestantes hipertensas crônicas, a reserva funcional da placenta tenha relação direta com seu "status vascular" em termos de comprometimento anatômico e de modificações mediadas pelo trofoblasto.

Estes aspectos também devem ser considerados em termos de planejamento terapêutico para estas pacientes. Não há consenso na literatura em relaçãa a esta questão, sendo que atualmente não existem respostas definitivas em relação aos riscos e benefícios do uso de hipotensores em gestantes com HAC leve ou moderada ${ }^{7.8}$.

A análise morfológica ou através de marcadores histoquímicos dos vasos da placenta e do leito placentário têm avanç̧ado em busca da compreensão de mecanismos complexos que modulam a invasão trofoblástica e a remodelação dos vasos deste território durante a gestação. Autores pioneiros, como Dixon e Robertson (1958), ${ }^{9}$ inauguraram a técnica da biópsia do leito placentário, procedimento que permite estudar a decídua basal e o miométrio subjacente

Vários estudos seguindo essa metodologia descreveram alterações morfológicas das artérias uteroplacentárias quando intercorrente a HAC ${ }^{10,11}, 1,12,13,14$. Porém, não se encontra nestes trabalhos a 
preocupação de correlacionar seus achados segundo as características clínicas maternas, verificando-se uma tendência na descrição de casos com maior gravidade clínica. Assim, consideramos pertinente estudar as características dos vasos do leito placentário estratificando estes achados segundo a expressão clínica da pressão arterial.

Acreditamos que o padrão morfológico vascular possa ser correlacionado com o desempenho perinatal exibido por grupos espeć́ficos de pacientes. Estes achados poderiam fornecer informações adicionais para estimativas de prognóstico perinatal de gestantes com características clínicas semelhantes, permitindo planejamento diferenciado para seu seguimento ao longo da gestação.

\section{Métodos}

O estudo foi tipo observacional, de caráter descritivo e comparativo, realizado a partir de biópsias do leito placentário em pacientes portadoras de HAC, submetidas ao parto cesariano no Departamento de Obstetrícia da Universidade Federal de São Paulo - Escola Paulista de Medicina ou no Serviço de Obstetrícia do Hospital Guilherme Álvaro, Faculdade de Ciências Médicas de Santos - UNILUS, Santos SP. O projeto foi aprovado pelo CEP de ambas as instituições, com os respectivos pareceres UNIFESP 369/04 e UNILUS 187.

Os fragmentos teciduais foram obtidos no período de junho de 2004 a julho de 2005. Foram relacionadas para o grupo controle, 25 gestantes normais (GC). O grupo de estudo foi constituído por pacientes portadoras de HAC selecionadas a partir dos critérios adotados pelo National High Blood Pressure Education Program (NHBPEP 2000), definindo essa condição quando a hipertensão é diagnosticada antes da vigésima semana de gestação ou quando aparece tardiamente na gravidez, mas não desaparece pós-parto (sem limitação de tempo) ${ }^{15}$.

A ausência de pré-eclâmpsia foi definida quando da verificação de ausência de proteinúria em urina de 24 horas (valores inferiores a 300 $\mathrm{mg}$ ). Nos casos em que as condições maternas e/ou fetais exigiam resolução antes das 24 horas necessárias para a coleta, foi utilizada a dosagem em amostras isoladas através de fitas reagentes.

O grupo de estudo foi dividido em três sub-grupos, segundo a classe de HAC, baseadas nos critérios de Lindheimer, Katz em 1984:16

- Hipertensão leve (HL), PAD 90mmHg e | - $100 \mathrm{mmHg}$

- Hipertensão moderada (HM), PAD $100 \mathrm{mmHg} \mid-110 \mathrm{mmHg}$.

- Hipertensão grave $(H G), P A D \geq \mid 10 \mathrm{mmHg}$.

Treze pacientes fizeram parte do grupo com hipertensão arterial leve (HL), I I integraram o grupo com hipertensão arterial moderada $(H M)$ e I I o grupo com hipertensão arterial crônica grave (HG).

O valor da pressão arterial aferida no momento da internação da paciente, independente do uso prévio de hipotensor, foi utilizado para classificar a intensidade da hipertensão (Tabela I). A medida ocorreu através de técnica padrão, com aparelho de coluna de mercúrio com manguito de $13 \mathrm{~cm}$, sendo utilizada tabela de correção segundo o perímetro do braço quando necessário ${ }^{17}$.

Foram incluídas gestantes com feto único e vivo, com idade gestacional igual ou superior a 28 semanas e placenta normalmente inserida. Foram excluídas pacientes que apresentaram: descolamento prematuro da placenta, acretismo placentário, malformações fetais, diabete melito, infecções agudas ou crônicas, colagenose,

\begin{tabular}{|c|c|c|c|}
\hline \multicolumn{4}{|c|}{$\begin{array}{c}\text { Tabela I - Medias e desvios padrão dos valores de pressão } \\
\text { arterial, segundo os grupos estudados }\end{array}$} \\
\hline Grupo & & Pressão sistólica & Pressão diastólica \\
\hline $\begin{array}{l}\text { Controle } \\
\mathrm{HL} \\
\mathrm{HM} \\
\mathrm{HG}\end{array}$ & $\begin{array}{l}\text { média } \\
\text { média } \\
\text { média } \\
\text { média }\end{array}$ & $\begin{array}{l}108,75(+/-13,93) \\
\mid 43,08(+/-7,23) \\
\mid 49,09(+/-\mid 6,40) \\
185,45(+/-\mid 8,64)\end{array}$ & $\begin{array}{l}71,46(+\mid-6,83) \\
90,77(+/-1,88) \\
100,45(+/-1,5 \mid) \\
116,36(+/-\mid 2,06)\end{array}$ \\
\hline
\end{tabular}

Pacientes com hipertensão grave.
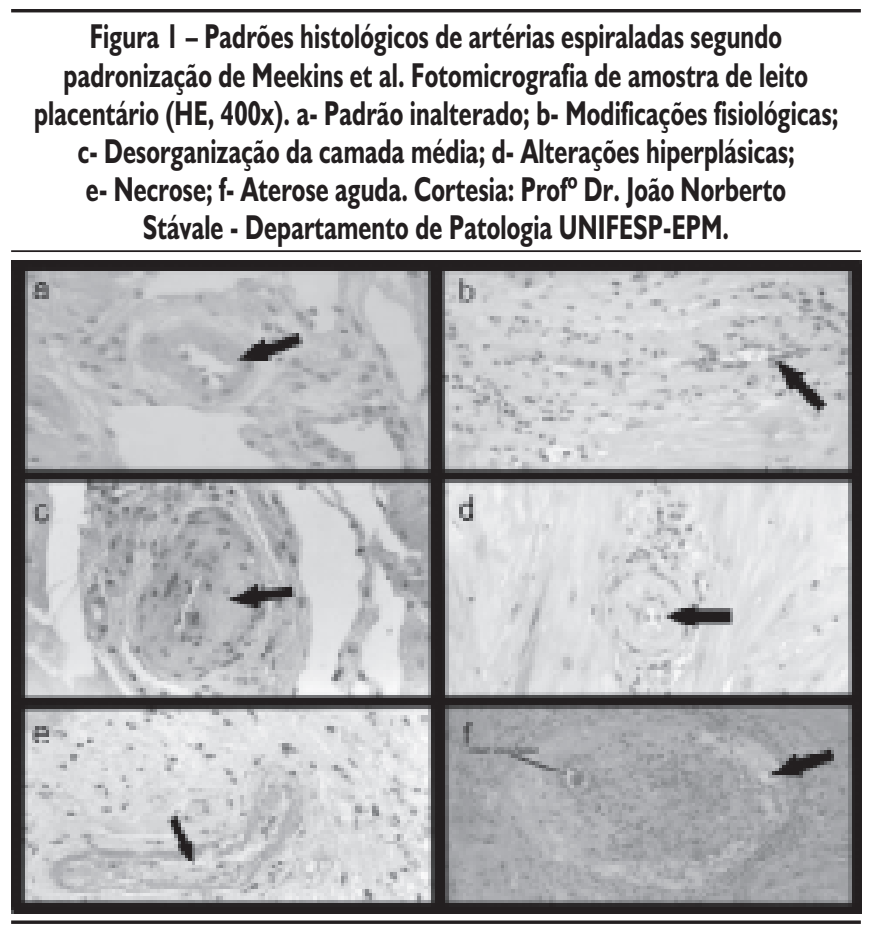

antecedentes de doenças tromboembólicas, tabagismo, pré-eclâmpsia sobreposta, aloimunização pelo fator $\mathrm{RH}$, alcoolismo, nefropatias e fragmentos teciduais do leito placentário que não preencheram os critérios de validação da amostra após avaliação histopatológical,18,19.

A biópsia foi realizada segundo técnica de Robertson et al. em 19861, 12, após dequitação, obtendo-se fragmento de cerca de I cm em extensão e profundidade na área mais próxima possível da porção central da implantação.

A amostra tecidual foi analisada por um único patologista que desconhecia o contexto clínico do caso. Os padrões histopatológicos (Figura I) foram classificados segundo os critérios de Meekins et al. ${ }^{18}$ em:

- Padrão inalterado: as artérias permaneceram com a morfologia semelhante à estrutura não gravídica (Figura la)

- Modificações fisiológicas: quando as artérias espiraladas apresentaram invasão trofoblástica endovascular (Figura Ib).

- Desorganização da camada média: caracterizada por fibrose e hialinização da parede vascular (Figura Ic) 


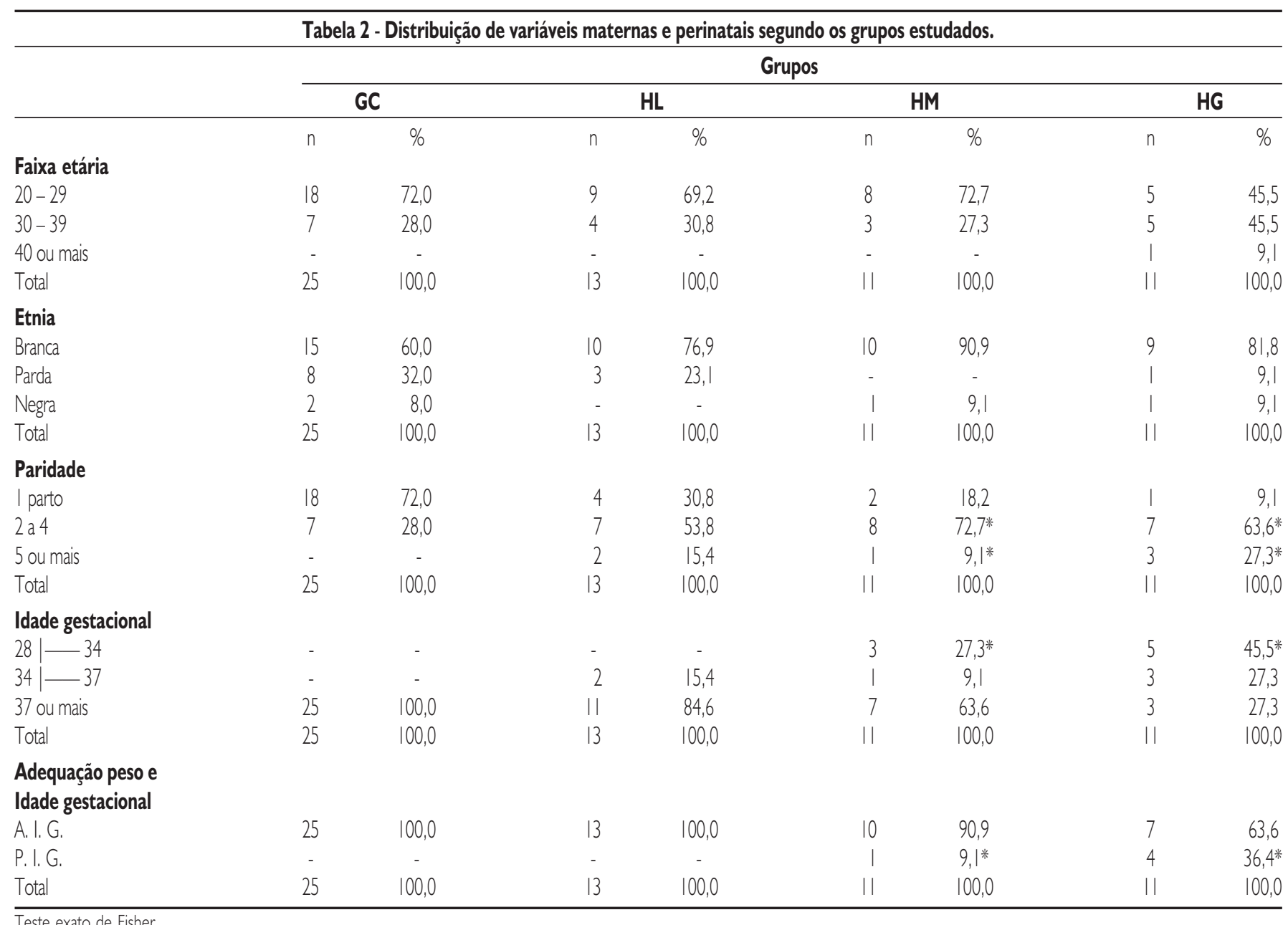

$p=0,00 I^{*}$

GC - Grupo Controle; HL - Pacientes com hipertensão leve; HM - Pacientes com hipertensão moderada; HG - Pacientes com hipertensão grave; A.I.G - Adequado para a idade gestacional; P.I.G - Pequeno para idade gestacional

- Alterações hiperplásicas das camadas íntima e média: definidas pelo espessamento da camada íntima e da camada média (Figura Id).

- Necrose caracterizada pela perda celular e eosinofilia intensa de todas as camadas (Figura le).

- Aterose aguda: determinada pela necrose fibrinóide, hialinização da parede vascular e infiltrado de histiócitos xantomizados na subíntima (Figura If).

Foram considerados como achados normais a presença de padrão normal e/ou modificações fisiológicas, enquanto que todos os outros padrões são classificados como patológicos. ${ }^{14} \mathrm{O}$ patologista verificou em cada fragmento a presença ou a ausência de cada um desses achados em pelo menos uma arteríola espiralada.

Foi calculado tamanho amostral de pelo menos II pacientes em cada grupo para um poder superior de $80 \%$ e erro alfa de $5 \%$. Para as comparações foi utilizado teste exato de Fisher. O nível de rejeição para a hipótese de nulidade foi fixado em valores menores ou iguais a 0,05 . Diferenças estatisticamente significantes foram assinaladas com asterisco.

\section{Resultados}

As características epidemiológicas e perinatais dos grupos estudados estão ilustradas na Tabela 2. Os grupos foram semelhantes quanto à idade e etnia, porém ocorreu maior número de multíparas, recém nascidos prematuros e pequenos para a idade gestacional (PIG) nos grupos $\mathrm{HM}$ e HG.

Os resultados referentes aos padrões histológicos (Tabela 3), identificaram diferenças significantes, pois os padrões patológicos ocorreram mais freqüentemente nos grupos $\mathrm{HM}$ e $\mathrm{HG}$, enquanto que os padrões normais foram encontrados com maior freqüência nos grupos GC e HL.

As modificações fisiológicas foram mais comuns nos grupos GC e HL,sendo que os padrões patológicos ocorreram de forma significativa nos grupos HM e HG. Dentre estes, foram observadas apenas desorganização da camada média e alterações hiperplásicas. A correlação entre os resultados ilustrados nas Tabelas 2 e 3 associa nível mais elevado da pressão arterial, presença significativa de padrões patológicos nas artérias espiraladas e pior prognóstico perinatal. 
Tabela 3 - Distribuição de achados histológicos em arteríolas espiraladas ${ }^{19} \mathrm{em}$ amostras de leito placentário, segundo classes de hipertensão arterial crônica

Grupos

\begin{tabular}{|c|c|c|c|c|c|c|c|}
\hline \multicolumn{2}{|c|}{ Controle } & \multicolumn{2}{|c|}{$\mathrm{HL}$} & \multicolumn{2}{|c|}{ HM } & \multicolumn{2}{|c|}{ HG } \\
\hline$n$ & $\%$ & $\mathrm{n}$ & $\%$ & $\mathrm{n}$ & $\%$ & $n$ & $\%$ \\
\hline
\end{tabular}

\section{Padrão}

\section{Normal}

Patológico

Total

Desorganização

\section{da camada média}

Ausente

Presente

Total

Alterações

\section{hiperplásicas}

Ausente

Presente

Total

$\begin{array}{cc}24 & 96,0 \\ 1 & 4,0 \\ 25 & 100,0\end{array}$

100,0

$\begin{array}{cc}12 & 92,3 \\ 1 & 7,7 \\ 13 & 100,0\end{array}$

100,0

2,3
7,7
00,0

3

$\begin{array}{cc}5 & 45,5 \\ 6 & 54,5 * \\ 11 & 100,0\end{array}$

$\begin{array}{ccc}96,0 & 12 & 92,3 \\ 4,0 & 1 & 7,7 \\ 100,0 & 13 & 100,0\end{array}$

0

$\begin{array}{cc}7 & 63,6 \\ 4 & 36,4 * \\ 11 & 100,0\end{array}$

$\begin{array}{cc}4 & 36,4 \\ 7 & 63,6 * \\ 11 & 100,0\end{array}$

36,4 100,0

(n)

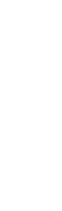

Teste exato de Fisher

$p=0,\left.00\right|^{*}$

HL - Pacientes com hipertensão leve; HM - Pacientes com hipertensão moderada; HG - Pacientes com hipertensão grave;

\section{Discussão}

Hipertensão arterial crônica é uma das intercorrências clínicas mais comuns na gestação, acarretando sérios problemas maternos e perinatais, incluindo a sobreposição de pré-eclâmpsia, restrição do crescimento fetal, prematuridade extrema, descolamento prematuro da placenta e morte materna e perinatal20,21,22.

Ainda que estes aspectos sejam amplamente reconhecidos, não existem estudos definitivos que possam esclarecer se existem riscos diferenciados entre as diversas classes de hipertensão. Em uma revisão sistemática, Ferrer et al, ${ }^{23}$ estimam que a hipertensão crônica triplique os riscos de mortalidade perinatal, dobre os riscos de descolamento da placenta e eleva as chances de restrição do crescimento fetal. Apesar dos riscos em hipertensas leves serem aparentemente menores quando comparados em hipertensas graves, a exata magnitude desta diferença não pode ser estimada a partir das evidências disponíveis atualmente.

Assim sendo, acreditamos que a observação das condições do leito placentário em gestantes portadoras de HAC possa fornecer subsídios adicionais para esta questão. Nossos achados identificaram uma relação positiva entre a presença de lesões vasculares crônicas em vasos da decídua e a condição clínica da paciente. Ou seja, quanto menor os níveis de hipertensão arterial, mais semelhante às condições vasculares em relação aos controles normais. Proporcionalmente, mais lesões foram identificadas nas pacientes com maiores níveis de pressão arterial.

Da mesma forma, verificamos que os resultados perinatais de pacientes normais e portadoras de formas leves de hipertensão foram semelhantes e com prognóstico significativamente melhor em relação as pacientes com pressão arterial moderada ou grave. Tais achados permitem supor que ainda que ocorra alguma alteração na estrutura vascular de hipertensas leves, estas não foram suficientes para afetar a reserva funcional da placenta, sendo que os resultados perinatais seriam influenciados na medida do agravamento destas alterações.

Em nosso estudo não foi identificado nas artérias avaliadas a presença de necrose e aterose aguda. Estas características diferem dos achados descritos por Mesquita et al, ao estudar a morfologia do leito placentário em pacientes portadoras de descolamento prematuro da placenta. Esta divergência de resultados talvez esteja relacionada com a gravidade clínica das pacientes estudadas pelos autores. Neste trabalho, o descolamento da placenta resultou provavelmente de um padrão vascular com comprometimento anatômico em fases mais avançadas quando comparados à população por nós estudada e identificava muitas vezes a sobreposição de pré-eclâmpsia, fato que não ocorreu em nossa população.

Aliás, em nossa metodologia, excluir a presença de pré-eclâmpsia teve como objetivo eliminar um viés de interpretação comum em trabalhos que avaliam o prognóstico de pacientes com HAC, uma vez que a vasoconstricção induzida pela pré-eclâmpsia tem importante parcela de responsabilidade nos resultados maternos e perinatais destas pacientes. Assim, os resultados obtidos em nossa casuística podem ser atribuídos exclusivamente ao padrão basal da estrutura vascular induzida apenas pela doença crônica.

Outro aspecto que merece destaque em nossos achados é a observação da presença de adaptação fisiológica das arteríolas espiraladas mesmo em gestantes com HAC. Tais achados são compatíveis com a observação clínica, em que é possível verificar que freqüentemente pacientes portadoras de HAC exibem redução de sua pressão arterial basal pré-gestacional ao longo da gestação. Em nosso 
estudo, verificamos que a ampla maioria $(92,3 \%$ ) das pacientes $\mathrm{HL}$, e cerca da metade das HM, apresentaram padrões morfológicos normais caracterizados por modificações fisiológicas dos vasos estudados, o que ocorreu em menor proporção nas pacientes HG.

Esta correlação poderia justificar o desempenho clínico e perinatal favorável de pacientes que exibem clinicamente esta redução da pressão arterial, fornecendo também algum subsídio para a decisão relativa à manutenção ou não de medicamentos hipotensores utilizados previamente à gestação.

Ainda em relaçãão ao uso de hipotensores, este não foi motivo de preocupação em nosso trabalho. Tal decisão foi baseada nos achados do trabalho de Meekins et al. ${ }^{18}$, que utilizando metodologia semeIhante, compararam os achados morfológicos das arteríolas espiraladas segundo o uso ou não de medicamentos hipotensores. Os autores concluíram que o uso destas drogas não foi capaz de ampliar a ocorrência de modificações adaptativas ao estado gravídico e nem atenuar os aspectos morfológicos deste território vascular.

Nossos achados são concordes com estes autores, ou seja, o desempenho perinatal em gestantes portadores de HAC parece estar relacionado com a capacidade de adaptação circulatória conseqüente às modificações fisiológicas da gravidez mediadas pela invasão trofoblástica, sendo que na medida que as lesões vasculares progridem, ocorre maior dificuldade para a instalação de processos adaptativos que garantam as necessidades para o desenvolvimento fetal.

\section{Conclusão}

Além da questão relativa ao prognóstico perinatal destas pacientes, ao se considerar a correlação anatomoclínica originada em nosso trabalho e no de outros autores, é razoável supor que o uso de hipotensores em pacientes hipertensas leves e mesmo em moderadas, não resultará em ganhos expressivos em termos de prognóstico perinatal. Estas informaç̧ões apoiariam por ora a opção por não utilizar hipotensores nestas pacientes. Conclusões definitivas dependem de ensaios clínicos controlados.

\section{Conflito de interesse: não há}

\section{SUMMARY}

\section{Morphology OF tHe VASCULAR PLACENTAL BED IN CHRONIC ARTERIAL HYPERTENSION}

OBIECTNES. To analyze histopathological patterns of placental bed arteries in pregnancies complicated by chronic arterial hypertension. Alterations were considered according to clinical classification of the hypertensive disorders as mild(MG); moderate(MoG) and severe(SG) for comparison with uncomplicated pregnancies, control group (CG).

METHODS. Placental bed biopsy was performed in 60 pregnantwomen; the study group was comprised of pregnant women with hypertension, subdivided in / 3 with severe chronichypertension (CH), I with moderate $\mathrm{CH}$ and I I with mild CH, and results were compared to 25 placental bed biopsies from uncomplicated pregnancies. All the pregnant women had a gestational age of at least 28 weeks of gestation with a live fetus and were submitted to cesarean section. Hypertension was considered mild with diastolic blood pressure (DBP) 90 l? $100 \mathrm{mmHg}$, moderate DBP 100 I?
$110 \mathrm{mmHg}$ and severe $D B P=110 \mathrm{mmHg}$. Placental bed variables selected for histological analysis were: unaltered patterns, physiological changes, medial layer disorganization, medial and intimal hyperplasic changes, acute necrosis and atherosis.

RESULTS. In cases with SG and MoG there was predominance of abnormal histophysiological findings: medial layer disorganization and hyperplasic changes, with a statistically significant difference when compared to MG and CG. Alteration in the medial layer was observed in these cases. The normal pattern, unaltered patterns and physiologic changes were more frequent in CG and MG. Physiological changes were the most usual finding, further, there was no acute necrosis or atherosis.

CONCLUSION. I. Abnormal histophysiologicalfindingswere predominant in hypertensive pregnant women compared to the normotensive ones; 2. These patterns were more frequent, according to the severity of the hypertensive disorders: Severe, Moderate and Mild; 3. More significant abnormal findings were a change in the medial layer, mainly in the group with severe hypertensive disorders; 4. The groups with moderate and severe hypertensive disorders when compared had similar results; 5 . The normal histological pattern was higher in the control group and patients with mild hypertensive disorders and disclosed a similarity between them 6. The most usual pattern was physiologic changes, which was more frequent in the control group. [Rev Assoc Med Bras 2008; 54(6): 537-42]

KEY wORDs: Chronic hypertension. Placental bed. Pregnancy.

\section{RefERÊNCIAS}

I. Robertson WB, Khong TY, Brosens I, De Wolf F, Sheppard BL, Bonnar J. The placental bed biopsy: review from three European centers. Am J Obstet Gynaecol. 1986; I 55(2):40 I - 12.

2. Ribeiro MD, Ribeiro $A B$, Stabile Neto $C$, Anção MS, Saragoça MA, Ramos OL, et al. Prevalência de hipertensão arterial na força de trabalho na Grande São Paulo: influência da idade, sexo e grupo etário. AMB Rev Assoc Med Bras. 1982; 28: 209-II

3. Hajjar I, Kotchen T. Trends in Prevalence, Awareness, Treatment, and Control of Hypertension in the United States, 1988-2000. JAMA 2003;290(2): 199-206

4. Sass N, Moron AF, El-Kadre D, Camano L, Almeida PAM. Contribuição ao estudo da gestação em portadores de hipertensão arterial crônica. Rev Paul Med. 1990; I08(6):261-5.

5. Kearney PM, Whelton M, Reynolds K, Muntner P, Whelton PK, He J. Global burden of hipertension: analysis of worldwide data. Lancet 2005; 365(9455): $217-23$.

6. Kearney PM, Whelton M, Reynolds K, Whelton PK, He J. Worldwide prevalence of hypertension: a systematic review. J Hypertens 2004; 22(I): II-9.

7. Sass N, Santos JFK, Atallah AN, Camano L. Hipertensão arterial crônica leve e moderada na gravidez: práticas terapêuticas baseadas em evidências. Femina. 2002;30(7):449-53.

8. Cunningham FG, Leveno KJ, Bloom SL, Hauth JC, Gisltrap III LC, Wenstrom KD. Chronic Hypertension. In: Cunningham FG, Leveno KJ, Bloom SL, Hauth JC, Gisltrap LC, Wenstrom KD. William's Obstetrics. 22th ed. New York: Mc-Graw-Hill; 2005. p. 1043-54.

9. Dixon HG \& Robertson WB. A study of the vessels of the placental bed in normotensive and hypertensive women. J Obstet Gynecol Br Emp. 1958;65(5):803-9.

10. Pijnenborg R. The placental bed. Hypertens Pregnancy 1996; 15:7-23

I I. Robertson WB, Brosens I, Dixon HG. The pathological response of the vessels of the placental bed to hypertensive pregnancy. J Pathol Bacteriol. 1967;93(2):581-92. 
12. Voigt HJ, Becker V. Doppler flow measurements and histomorphology of the placental bed in uteroplacental insufficiency. J Perinat Med. 1992;20(2): 139-47.

13. Ramos JG, Martins-costa S, Edelweiss MI, Costa CA. Placental bed lesions and infant birth weight in hypertensive pregnant women. Bras J Med Biol Res. 1995;28(4):447-55.

14. Mesquita MRS, Sass N, Stavalle JN, Camano L. O leito placentário no descolamento prematuro da placenta. Rev Bras Ginecol Obstet. 2003;25(8):585-91.

15. National High Blood Pressure Education Program. Report of the National High Blood Pressure Education Program Working Group on High Blood Pressure in Pregnancy. Am J Obstet Gynecol. 2000; I 83(I):SI-S22

16. Lindheimer MD, Katz Al. Hypertension in pregnancy. N Engl J Med. 1985;3 I3(I I):675-80.

17. Maxwell MH, Waks AU, Schroth PC, Karam M, Dornfeld LP. Error in blood-pressure measurement due to incorrect cuff size in obese patients. Lancet. 1982;2(8288):33-6.

18. Meekins JW, Pijnenborg R, Hanssens M, Mcfadyen IR, Van Assche FA. Spiral artery morphology in pregnancies complicated by chronic hypertension: the relation to superimposed preeclampsia. 1995; I 4(I):67-80.
19. Gerretsen G, Huisjes HJ, Hardonk MJ, Elema JD. Trophoblast alterations in the placental bed in relation to physiological changes in spiral arteries. Br J Obstet Gynaecol. 1983; 90(I):34-9

20. Ojeda NB, Grigore D, Alexander BT. Intrauterine growth restriction: fetal programming of hypertension and kidney disease. Adv Chronic Kidney Dis. 2008; I 5(2): I $01-6$.

21. Chappell LC, Enye S, Seed P, Briley AL, Poston L, Shennan AH. Adverse perinatal outcomes and risk factors for preeclampsia in women with chronic hypertension: a prospective study. Hypertension (Hypertension). 2008;5। (4): I002-9.

22. Gilbert WM, Young AL, Danielsen B. Pregnancy outcomes in women with chronic hypertension: a population-based study. J Reprod Med (The Journal of reproductive medicine). 2007;52( I I): I046-5 I.

23. Ferrer RL, Sibai BM, Mulrow CD, Chiquette E, Stevens KR, Cornell J. Management of mild chronic hypertension during pregnancy: a review. Obstet Gynecol 2000;96:849-60.

$$
\text { Artigo recebido: 22/04/07 }
$$

